\title{
Exploitation of Host Epithelial Signaling Networks by Respiratory Bacterial Pathogens
}

\author{
Jian-Dong $\mathrm{Li}^{1,2, *}$ \\ ${ }^{1}$ Gonda Department of Cell and Molecular Biology, House Ear Institute, \\ 2100 West Third Street, Los Angeles, CA 90057, USA \\ ${ }^{2}$ Department of Otolaryngology, University of Southern California, Los Angeles, CA 90057, USA
}

Received September 30, 2002

\begin{abstract}
Although tremendous effort has been put towards identifying the surface molecules of nontypeable Haemophilus influenzae (NTHi) for vaccine development over the past decades, it is only recently that we have begun to appreciate the intricate host epithelial signaling networks activated by NTHi, an important human pathogen causing respiratory infections. From what has been reported, it is evident that NTHi activates multiple signaling pathways in host epithelial cells that, in turn, inadvertently contribute to the pathogenesis. Among those signaling pathways, activation of NF- $\kappa$ B leads to up-regulation of IL- $1 \beta$, IL-8 and TNF- $\alpha$, mucin MUC2 and Tolllike receptor 2 (TLR2), whereas activation of p38 MAP kinase mediates not only up-regulation of inflammatory mediators and mucin MUC5AC but also down-regulation of TLR2. Interestingly, NTHi-induced activation of the PI3K-Akt pathway, however, leads to inhibition of p38 mitogen-activated protein (MAP) kinase. Moreover, the TGF- $\beta$-Smad signaling pathway cooperates with NF- $\kappa \mathrm{B}$ to mediate up-regulation of mucin $M U C 2$. Finally, glucocorticoids synergistically enhance NTHi-induced TLR2 expression via specific up-regulation of the MAP kinase phosphatase- 1 that, in turn, leads to inactivation of p38 MAP kinase, the negative regulator for TLR2 expression. These studies may bring new insights into the molecular pathogenesis of NTHi-induced infections and open up novel therapeutic targets for these diseases.
\end{abstract}

Keywrods: chronic obstructive pulmonary disease, glucocorticoids, p38 MAPK, nuclear factor- $\kappa \mathrm{B}$, nontypeable Haemophilus influenzae

\section{Introduction}

Recently, interest in exploitation of host cell signaling machinery by bacteria has increased because of the dramatic increase in antibiotic-resistant strains, the emergence of new pathogens and the resurgence of old ones. Bacterial pathogens have evolved many sophisticated ways to subvert normal host cellular responses. One such subversive pathogen is the gram-negative bacterium nontypeable Haemophilus influenzae (NTHi), which exemplifies an important, as yet underexplored, human respiratory pathogen in both children and adults (1). In children, it causes otitis media (OM), the most

*Corresponding author (affiliation \#1). FAX: +1-213-273-8088

E-mail: jdli@hei.org

Invited article common childhood infection and the leading cause of conductive hearing loss $(2,3)$, while in adults, it exacerbates chronic obstructive pulmonary diseases (COPD), the fourth leading cause of death in the United States $(4,5)$. Despite the need for prophylactic measures, development of a vaccine for preventing NTHi infections has been difficult and still remains a great challenge (1). Moreover, inappropriate antibiotic treatment contributes to the worldwide emergence of antibioticresistant strains of NTHi. Therefore, alternative therapeutic strategies are urgently needed to counter NTHi infections that are previously treated by conventional antibiotics. Development of these novel therapeutic reagents, however, requires a full understanding of the molecular mechanisms, especially the signaling mechanisms underlying the NTHi infections. 
$\mathrm{NF}-\kappa \mathrm{B}$ plays a critical role in NTHi-induced inflammation and up-regulation of mucin, hallmarks of COPD and otitis media

Although much work has been done on identifying the surface molecules of NTHi for vaccine development $(1,2)$, the molecular mechanisms including the signaling mechanisms underlying the pathogenesis of NTHi infections still remain poorly understood. Like most other gram-negative bacterial infections, both otitis media and chronic obstructive pulmonary diseases are characterized by inflammation, which is mainly mediated by inflammatory cytokines and chemokines such as interleukin-1 $\beta$ (IL-1 $\beta$ ), interleukin-8 (IL-8) and tumor necrosis factor- $\alpha$ (TNF- $\alpha)(6-8)$. Among a variety of transcription regulators, nuclear factor $\kappa \mathrm{B}(\mathrm{NF}-\kappa \mathrm{B})$ has been shown to play a critical role in regulating the expression of large numbers of genes including cytokines, chemokines and other mediators involved in inflammatory responses (9).

$\mathrm{NF}-\kappa \mathrm{B}$ is a dimeric transcription factor that is composed of p50 (NF- $\kappa \mathrm{B} 1)$ and p65 (RelA) subunits (9). In resting cells, NF- $\kappa \mathrm{B}$ is retained in the cytoplasm but enters the nucleus in response to various stimuli including bacterial infections. Activation of NF- $\kappa \mathrm{B}$ is controlled by an inhibitory subunit, $\mathrm{I} \kappa \mathrm{B}$, which retains $\mathrm{NF}-\kappa \mathrm{B}$ in the cytoplasm. The activation of NF- $\kappa \mathrm{B}$ requires sequential phosphorylation, ubiquitination, and degradation of $\mathrm{I} \kappa \mathrm{B}$ as well as consequent exposure of a nuclear localization signal on $\mathrm{NF}-\kappa \mathrm{B}$ molecule. Multiple kinases have been shown to phosphorylate $\mathrm{I} \kappa \mathrm{B}$ at specific amino-terminal serine residues $(10,11)$. The most well studied kinases are I $\kappa$ B kinases, IKK $\alpha$ (or IKK-1) and IKK $\beta$ (or IKK-2) (9). There is also strong evidence that IKK $\alpha$ and IKK $\beta$ are themselves phosphorylated and activated by one or more upstream activating kinases. One such upstream kinase, NF- $\kappa \mathrm{B}$ inducing kinase (NIK), was recently identified (9). Phosphorylation of $\mathrm{I} \kappa \mathrm{B}$ by the IKKs pathway will eventually lead to the nuclear translocation of NF- $\kappa \mathrm{B}$, which, in turn, activates expression of target genes in the nucleus.

Based on the essential involvement of NF- $\kappa \mathrm{B}$ in inflammatory responses, activation of NF- $\kappa \mathrm{B}$ by NTHi has been recently fully explored. Interestingly, NTHi appears to strongly activate NF- $\kappa$ B in human epithelial cells via two distinct signaling pathways, NF- $\kappa$ B translocation-dependent and independent pathways (12). The $\mathrm{NF}-\kappa \mathrm{B}$ translocation-dependent pathway involves activation of the NIK-IKK $\alpha / \beta$ complex, leading to $\mathrm{I} \kappa \mathrm{B} \alpha$ phosphorylation and degradation, while the NF- $\kappa \mathrm{B}$ translocation-independent pathway involves activation of the MKK3/6-p38 MAP kinase pathway. Bifurcation of NTHi-induced NIK-IKK $\alpha / \beta$-I $\kappa \mathrm{B} \alpha$ and MKK3/6- p38 MAP kinase pathways may occur at TGF- $\beta$-activated kinase I (TAK1). Furthermore, Toll-like receptor 2 (TLR2) is required for NTHi-induced NF- $\kappa \mathrm{B}$ activation. In addition, several key inflammatory mediators including IL- $1 \beta$, IL- 8 and TNF- $\alpha$ are up-regulated by NTHi. Finally, P6, a $16-\mathrm{kDa}$ lipoprotein highly conserved in the outer membrane of all NTHi and H. influenzae type $b$ strains, appears to also activate NF- $\kappa$ B via similar signaling pathways. Taken together, these results demonstrate that NTHi activates NF- $\kappa$ B via a TLR2-TAK1dependent NIK-IKK $\alpha / \beta$-I $\kappa \mathrm{B} \alpha$ and MKK3/6-p38 MAP kinase signaling pathways that, in turn, leads to up-regulation of key inflammatory mediators (Fig. 1). In addition to its role in regulating inflammatory responses, activation of NF- $\kappa \mathrm{B}$ via a NIK-IKK $\alpha / \beta$-I $\kappa \mathrm{B} \alpha$ signaling pathway has been also shown to mediate NTHiinduced up-regulation of mucin $M U C 2$ and TLR2 (13, 14). These studies may lead to new therapeutic intervention for NTHi-induced infections including otitis media and chronic obstructive pulmonary diseases.

\section{p38 MAP kinase also plays a key role in the patho- genesis of NTHi infections}

In addition to NF- $\kappa \mathrm{B}$ pathway p38 MAP kinase (mitogen-activated protein kinase) $(15,16)$ represents another key signaling module involved in mediating cellular stress responses during bacterial infections. p38 MAP kinase consists of 4 isoforms, $\alpha, \beta, \gamma$, and $\delta$. Together with its upstream MAP kinase kinases (MKKs), p38 MAP kinase comprises another important MAP kinase pathway (16). The p38 MAP kinase pathway is in many ways homologous to the classical extracellular signal-regulated kinases 1 and 2 (ERK1/2) pathway, but it appears to be activated by distinct upstream kinases, MKK3, MKK4 and MKK6, and is stimulated by a variety of cellular stresses including osmotic shock, inflammatory cytokines and LPS (17). Activated p38 MAP kinase, either directly activates transcription factors such as TCF, ATF2, and Max, or it activates downstream protein kinases such as p38-regulated/activated protein kinase (PRAK), which, in turn, activates the transcription factors $(18,19)$. Development of p38 MAP kinase inhibitors provides a powerful tool for studying the role of $\mathrm{p} 38$ in stress-induced cellular responses. Among the inhibitors, SB203580 has been shown to selectively inhibit p38 $\alpha$ and $\beta$, but not $\gamma$ or $\delta$-nor any other member of the MAP kinase family $(20,21)$. Because of its high selectivity, SB203580 has been widely used in cellular assays as a probe for the involvement of p38 (22). Moreover, tremendous effort is being put towards developing novel therapeutic agents that are based on the structure of SB203580 (22). Taking 
advantage of SB203580 and available dominant-negative mutant constructs of $\mathrm{p} 38$, it has been shown recently that activation of $\mathrm{p} 38$ MAP kinase $(\alpha$ or $\beta$ ) is required for NTHi-induced MUC5AC transcriptional activation (23) (Fig. 2). In sharp contrast to its positive involvement in NTHi-induced mucin MUC5AC transcription, the MKK3/6-p38 $\alpha / \beta$ signaling pathway, however, serves as a negative regulator for NTHi-induced TLR2 transcription (14). These results thus indicate that NTHi also strongly activates p38 MAP kinase in addition to NF- $\kappa \mathrm{B}$, which, in turn, leads to differential transcriptional regulation of host defense genes in the pathogenesis of NTHi infections.

\section{Phosphoinositide 3-kinase (PI3-kinase)-Akt signaling pathway acts as a negative regulator for p38 MAP kinase}

Besides NF- $\kappa$ B and p38 MAP kinase pathways, phosphoinositide 3-kinase (PI3-kinase) represents another major signaling transducer involved in a variety of cellular responses. It is a heterodimer consisting of $\mathrm{p} 85$, the regulatory subunit, and $\mathrm{p} 110$, the catalytic subunit. Activation of PI3-kinase catalyzes the phosphorylation of phosphatidylinositol. The phosphorylated lipids bind to Akt, a serine-threonine kinase, resulting in membrane localization and a conformational change of Akt. This allows Akt to be phosphorylated and activated to mediate a variety of cellular responses such as protection of cells from apoptosis (24) and induction of NF- $\kappa \mathrm{B}$ (25). There is also evidence that PI3-kinase is involved in bacterial pathogenesis (26). Because of the importance of PI3-kinase-Akt in cellular responses as well as in bacterial pathogenesis, efforts have been put towards determining the potential involvement of PI3-kinase-Akt in NTHi-induced MUC5AC transcription. Interestingly, the PI3-kinase-Akt signaling pathway also appears to be activated by NTHi, which, however, leads to down-regulation of $\mathrm{p} 38$ MAP kinase activity (23) (Fig. 3). Negative cross-talk has been established by previous studies between the PI3-kinase-Akt pathway and MAP kinases including ERK and the c-jun $\mathrm{NH}_{2}$-terminal kinase (JNK) (27). Whether or not there is also negative interaction between PI3-kinase-Akt and p38 MAP kinase has remained unclear. Recently, a report by Gratton et al. showed that blockade of PI3kinase-Akt led to enhanced vascular endothelial growth factor (VEGF) activation of p38 MAP kinase (28). However, little is known about the involvement of this negative cross-talk in bacterial pathogenesis as well as in mucin gene regulation. In the case of NTHi infections, PI3-kinase-Akt serves as an inhibitory signaling pathway in NTH-induced MUC5AC transcription via a negative cross-talk with p38 MAP kinase. Although it has been shown that inhibition of the PI3-kinase-Akt signaling by wortmannin enhanced, whereas activation of PI3-kinase-Akt by overexpression of an activated form of p110 attenuated, NTHi-induced activation of p38 MAP kinase, it is difficult to rule out the possibility that the PI3-kinase-Akt pathway may interact with the upstream p38 MAP kinases such as MAP kinase kinase 3 and 6 (MKK3/6) based on current report. It is also unclear whether a direct physical interaction between PI3-kinase-Akt and MKK3/6-p38 MAP kinase is involved in this cross talk. These questions will be addressed in our future studies.

\section{TGF- $\beta$ signaling pathway cooperates with NF- $\kappa$ B to mediate NTHi-induced host defensive response}

The TGF- $\beta$-Smad pathway represents another important signaling pathway participating in regulation of diverse biological processes, including cell proliferation, differentiation, death, and inflammatory and immune responses $(29-35)$. The TGF- $\beta$ superfamily is a large group of secreted growth factors of which three subgroups have been defined: the TGF- $\beta \mathrm{s}$, activins and bone morphonegetic proteins (BMPs) $(32,34)$. TGF- $\beta$ initiates signaling through the ligand-dependent activation of a heteromeric complex of type II and type I receptors. The type II receptor kinase then phosphorylates the type I receptor in a conserved glycine-serine domain (GS domain), resulting in activation of the type I receptor. The activated type I receptor subsequently recognizes and phosphorylates the Smad subgroup known as receptor-activated Smads (R-Smad), including Smad 2 and 3. This causes dissociation of R-Smad from the receptor, stimulates the assembly of a heteromeric complex between the phosphorylated R-Smad and the Co-Smad, Smad 4, and then induces the translocation of the Smad complex to the nucleus, where the Smad complex regulates the expression of target genes (30, 32). In addition to its direct interaction with Smad DNAbinding element (SBE), growing evidence suggests that Smads also regulate gene transcription by direct interaction and functional cooperation with other transcription factors, such as NF- $\kappa \mathrm{B}(36-38)$. Despite its important role in regulation of diverse biological processes, it is still unclear if activation of the TGF- $\beta$-Smad signaling pathway also mediates up-regulation of mucin, a primary host innate defensive response to bacteria.

Because of the important role of NF- $\kappa \mathrm{B}$ and TGF- $\beta$ signaling in mediating diverse cellular responses as well as the reported functional cooperation between NF- $\kappa \mathrm{B}$ and TGF- $\beta$-Smad, it is logical that the TGF- $\beta$-Smad signaling pathway cooperates with NF- $\kappa \mathrm{B}$ to mediate 


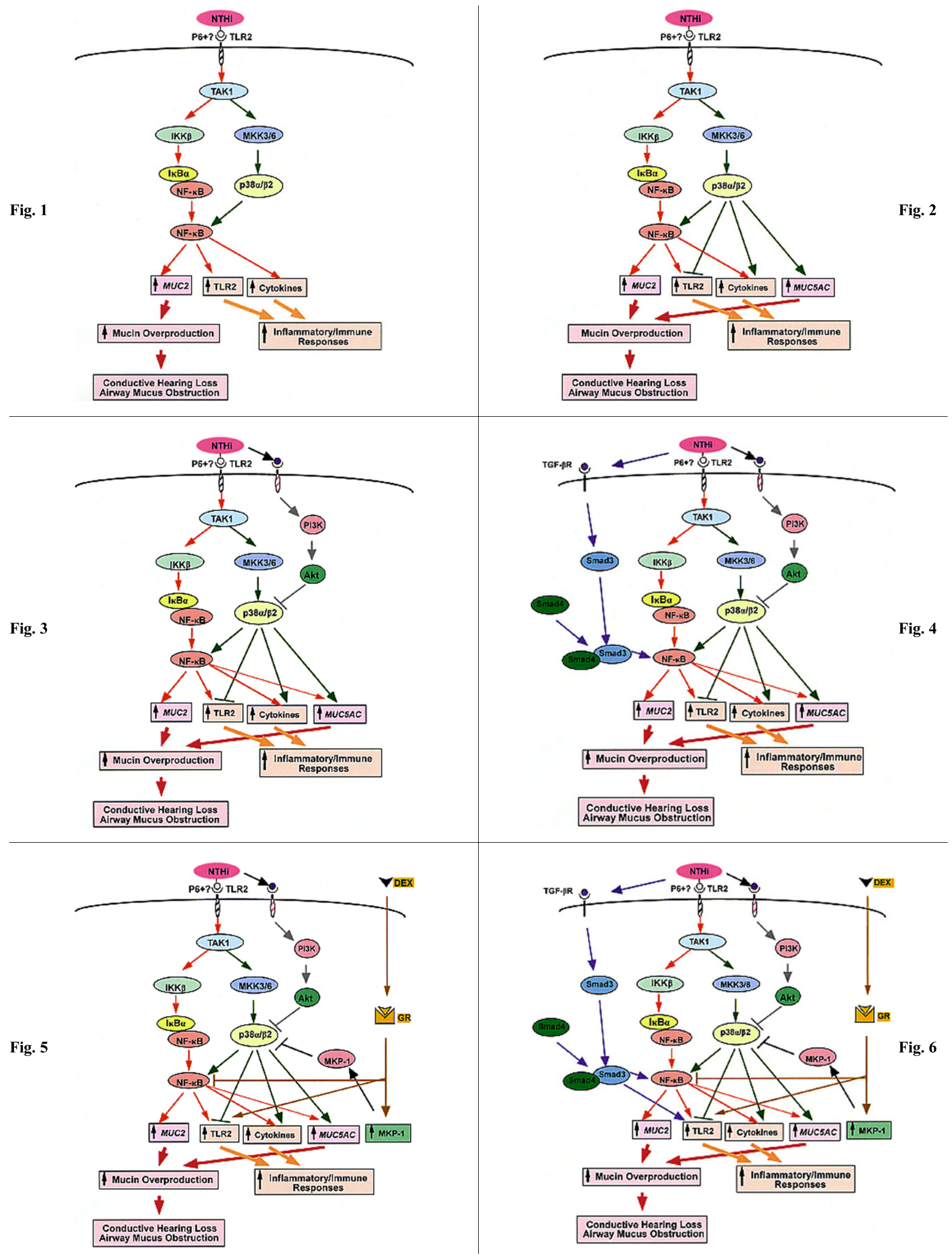


up-regulation of $M U C 2$ mucin transcription in response to NTHi infections in human epithelial cells. Indeed, activation of TGF- $\beta$ receptor-Smad3/4 signaling, together with TLR2-MyD88-TAK1-NIK-IKK $\beta / \gamma$-I $\kappa \mathrm{B} \alpha$ dependent activation of $\mathrm{NF}-\kappa \mathrm{B}$, has been recently shown to mediate NTHi-induced MUC2 mucin transcription (13). These findings provided direct evidence, for the first time, that the bacterium NTHi uses TGF- $\beta$ Smad pathway for transducing signals into nucleus, at least in part, via an autocrine-independent mechanism, and that the functional cooperation between NF- $\kappa$ B and TGF- $\beta$ receptor-Smad is required for host defensive response to NTHi (Fig. 4). These studies may bring insights into the novel role of TGF- $\beta$-Smad signaling in bacterial pathogenesis and may lead to novel therapeutic intervention for otitis media and COPD.

\section{Orchestration and optimization of host immune and defense responses by glucocorticoids in NTHi infec- tions}

Despite the importance of glucocorticoids in suppressing immune and inflammatory responses, their role in enhancing host immune and defense response against invading bacterial pathogens is poorly understood (3943). Moreover, the molecular basis for the beneficial role of glucocorticoids in the treatment of bacterial infections such as Haemophilus influenzae-induced bacterial meningitis also remains unclear. Recently, it was reported that glucocorticoids synergistically en- hance the expression of TLR2, an important member of the TLR family that has been shown to play a crucial role in host immune and defense response, as evidenced by the studies from knockout mice showing decreased survival of TLR2-deficient mice after infection with Gram-positive S. aureus $(14,44)$. Thus, it is likely that glucocorticoids may not only suppress but also enhance the host immune and defense response. In addition, our study may also provide a molecular basis for the explanation of the beneficial role of glucocorticoids in certain bacterial infections. What are the molecular mechanisms by which the glucocorticoid-mediated enhancement of TLR2 contributes to host immune and defense response? Given the fact that the TLR2 expression is low in unstimulated epithelial cells, the synergistic enhancement of NTHi-induced TLR2 expression by glucocorticoids will probably contribute to the accelerated immune response by epithelial cells as well as resensitization of epithelial cells to invading pathogens. If so, up-regulation of TLR2 may be one of the positive immune-regulatory mechanisms involved in glucocorticoid-mediated host defense against many bacterial pathogens. However, the signaling mechanisms underlying glucocorticoid-mediated synergistic enhancement of TLR2 are still unknown. Nor is the physiological relevance of TLR2 up-regulation known at present. Very recently, we showed that, glucocorticoids synergistically enhance the NTHi-induced TLR2 expression via specific up-regulation of the MAP kinase phosphatase-1 (MKP-1) (45-47), which, in turn, leads to dephos-

Fig. 1. Schematic representation of NTHi-induced signal transduction pathways involved in NF- $\kappa \mathrm{B}$ activation in human epithelial cells. IKK, I $\kappa$ B kinase; NTHi, nontypeable Haemophilus influenzae; MKK, MAP (mitogen-activated protein) kinase kinase; TAK1, TGF- $\beta$-activated kinase 1; TLR, Toll-like receptor.

Fig. 2. Schematic representation of NTHi-induced signal transduction pathways involved in p38 MAP kinase activation in human epithelial cells. IKK, I $\kappa$ B kinase; NTHi, nontypeable Haemophilus influenzae; MKK, MAP (kinase, mitogen-activated protein) kinase kinase; TAK1, TGF- $\beta$-activated kinase 1 ; TLR, Toll-like receptor.

Fig. 3. Schematic representation of NTHi-induced PI3-kinase-Akt signaling pathways involved in inhibition of p38 MAP kinase. IKK, I $\kappa \mathrm{B}$ kinase; NTHi, nontypeable Haemophilus influenzae; MKK, MAP (mitogen-activated protein) kinase kinase; PI3K: phosphoinositide 3-kinase; TAK1, TGF- $\beta$-activated kinase 1; TLR, Toll-like receptor.

Fig. 4. Schematic diagram showing that TGF- $\beta$ signaling pathway cooperates with NF- $\kappa \mathrm{B}$ to mediate NTHi-induced host defensive response. IKK, I $\kappa$ B kinase; NTHi, nontypeable Haemophilus influenzae; MKK, MAP (mitogen-activated protein) kinase kinase; TAK1, TGF- $\beta$-activated kinase 1; TGF- $\beta$ R: transforming growth factor- $\beta$-receptor; TLR, Toll-like receptor.

Fig. 5. Schematic representation of the signaling pathways involved in glucocorticoid-mediated enhancement of NTHi-induced TLR2 expression via MKP-1-dependent inhibition of p38 MAPK in human epithelial cells. DEX, dexamethasone; GR, glucocorticoids receptor; IKK, I $\kappa$ B kinase; NTHi, nontypeable Haemophilus influenzae; MKK, MAP (mitogen-activated protein) kinase kinase; MKP-1, MAP kinase phosphatase-1; TAK1, TGF- $\beta$ activated kinase 1; TLR, Toll-like receptor.

Fig. 6. Schematic representation of NTHi-induced host epithelial signaling networks in the pathogensis of NTHi infections. DEX, dexamethasone; GR, glucocorticoids receptor; NTHi, nontypeable Haemophilus influenzae; MKK, MAP (mitogen-activated protein) kinase kinase; MKP-1, MAP kinase phosphatase-1; TAK1, TGF- $\beta$-activated kinase 1; TGF- $\beta$ R: transforming growth factor- $\beta$ receptor; TLR, Toll-like receptor. 
phorylation and inactivation of p38 MAP kinase, the negative regulator for TLR2 expression (Fig. 5). Moreover, increased expression of TLR2 in epithelial cells also greatly enhance the NTHi-induced expression of several key cytokines including TNF- $\alpha$, IL- $\beta \beta$ and IL-8, thus demonstrating the physiological relevance of TLR2 up-regulation in bacterial pathogenesis. These studies, although rather unexpected, may provide novel insights into the role of glucocorticoids in orchestrating and optimizing host defense and innate immune responses and lead to novel therapeutic strategies for modulating innate immune and inflammatory responses for bacterial infections.

\section{Concluding remarks}

Although tremendous effort has been put towards identifying the surface molecules of NTHi for vaccine development over the past decades $(1,2)$, it is only recently that we have begun to appreciate the intricate host epithelial signaling networks activated by NTHi, an important human bacterial pathogen causing respiratory infections. From the studies that we have reviewed above, it is evident that NTHi activates multiple signaling pathways in host epithelial cells that, in turn, inadvertently contribute to the pathogenesis and disease outcome (Fig. 6). Among those signaling pathways activated by NTHi, activation of NF- $\kappa$ B via a TLR2-TAK1dependent NIK-IKK $\alpha / \beta$-I $\kappa \mathrm{B} \alpha$ leads to up-regulation of key inflammatory mediators including IL- $1 \beta$, IL-8 and TNF- $\alpha$, mucin MUC2 and TLR2 $(13,14)$, whereas activation of the MKK3/6-p38 MAP kinase signaling pathway mediates not only up-regulation of inflammatory mediators and mucin MUC5AC but also downregulation of TLR2. Interestingly, NTHi-induced activation of PI3K-Akt pathway, however, leads to negative regulation of p38 MAPK activity. Moreover, a functional cooperation between the TGF- $\beta$-Smad signaling pathway and NF- $\kappa \mathrm{B}$ appears to mediate up-regulation of MUC2 mucin transcription in response to NTHi infections. Finally, glucocorticoids synergistically enhance NTHi-induced TLR2 expression via specific upregulation of MKP-1 that, in turn, leads to dephosphorylation and inactivation of $\mathrm{p} 38$ MAP kinase, the negative regulator for TLR2 expression. This result, although rather unexpected, may provide novel insights into the role of glucocorticoids in orchestrating and optimizing host defense and innate immune responses. These studies may bring new insights into molecular pathogenesis of NTHi-induced infections and open up new therapeutic targets for these diseases. Future efforts will be focused on identifying the key therapeutic targets for the treatment of NTHi infections using a multidisciplinary approach including bacterial genetics and biochemistry, mammalian cell biology, molecular biology, immunology, and knockout mice.

\section{Acknowledgments}

I thank the members of my lab and collaborators for their scientific contributions and discussions. Work in my lab is supported by grants from NIH DC04562 (to J.D. Li) and NIH DC005843 (to J.D. Li).

\section{References}

1 Foxwell AR, Kyd JM and Cripps AW: Nontypeable Haemophilus influenzae: pathogenesis and prevention. Microbiol Mol Biol Rev 62, 294 - 308 (1998)

2 Murphy TF: Bacterial otitis media: pathogenetic considerations. Pediatr Infect Dis J 19, Suppl 5, S9 - S15 (2000)

3 Bluestone CD: Otitis media in children: to treat or not to treat? N Engl J Med 306, 1399 - 1404 (1982)

4 ATS Statement: Am J Respir Crit Care Med 152, Suppl 5, 78S 121S (1995)

5 Murphy TF: Haemophilus influenzae in chronic bronchitis. Semin Respir Infect 15, 41 - 51 (2000)

6 Bluestone CD: Clinical course, complications and sequelae of acute otitis media. Pediatr Infect Dis J 19, Suppl 5, S37 - S46 (2000)

7 Di Mango E, Ratner AJ, Bryan R, Tabibi S and Prince A: Activation of NF-kappaB by adherent Pseudomonas aeruginosa in normal and cystic fibrosis respiratory epithelial cells. J Clin Invest 101, 2598 - 2605 (1998)

8 Melhus A and Ryan AF: Expression of cytokine genes during pneumococcal and nontypeable Haemophilus influenzae acute otitis media in the rat. Infect Immun 68, $4024-4031$ (2000)

9 Hatada EN, Krappmann D and Scheidereit C: NF-kappaB and the innate immune response. Curr Opin Immunol 12, 52-58 (2000)

10 Stancovski I and Baltimore D: NF-kappaB activation: the I kappaB kinase revealed? Cell 91, 290 - 302 (1997)

11 Karin M: How NF-kappaB is activated: the role of the IkappaB kinase (IKK) complex. Oncogene 18, 6867 - 6874 (1999)

12 Shuto T, Xu H, Wang B, Han J, Kai H, Gu XX, Murphy T, Lim DJ and Li JD: Activation of NF-kappa B by nontypeable Hemophilus influenzae is mediated by toll-like receptor 2TAK1-dependent NIK-IKK alpha/beta-I kappa B alpha and MKK3/6-p38 MAP kinase signaling pathways in epithelial cells. Proc Natl Acad Sci USA 98, 8774 - 8779 (2001)

13 Jono H, Xu H, Kai H, Gum J, Kim YS, Feng XH and Li JD: Direct activation of TGF- $\beta$-Smad signaling by nontypeable Haemophilus influenzae mediates host defensive responses. J Biol Chem 277, 45547 - 45557 (2002)

14 Shuto T, Imasato I, Jono H, Xu H, Watanabe T, Kai H, Andalibi A, Linthicum F, Guan YL, Han J, Cato AC, Akira S, Lim DJ and Li JD: Glucocorticoids synergistically enhance nontypeable Haemophilus influenzae-induced Toll-like receptor 2 expression via a negative cross-talk with p38 MAP kinase. J Biol Chem 277, 17263 - 17270 (2002)

15 Schulze-Osthoff K, Ferrari D, Riehemann K and Wesselborg S: 
Regulation of NF-kappa B activation by MAP kinase cascades. Immunobiology 198, 35 - 49 (1997)

16 Ono K and Han J: The p38 signal transduction pathway: activation and function. Cell Signal 12, 1- 13 (2000)

17 New L and Han J: The p38 MAP kinase pathway and its biological function. Trends in Cardiovascular Medicine 8, $220-$ 228 (1998)

18 Han J, Jiang Y, Li Z, Kravchenko VV and Ulevitch RJ: MEF2C participates in inflammatory responses via p38 activation. Nature 386, 296 - 299 (1997)

19 New L, Jiang Y, Zhao M, Liu K, Flood L, Kato Y, Parry GC and Han J: PRAK, a novel protein kinase regulated by the p38 MAP kinase. EMBO J 17, 3371 - 3384 (1998)

20 Cuenda A, Rouse J, Doza YN, Meier R, Cohen P, Gallagher TF, Young PR and Lee JC: SB203580 is a specific inhibitor of a MAPK homologue which is stimulated by cellular stresses and interleukin-1. FEBS Lett 364, 229 - 233 (1995)

21 Young PR, McLaughlin MM, Kumar S, Adams JL and Lee JC: Pyridinyl imidazole inhibitors of $\mathrm{p} 38$ MAP kinase bind in the ATP site. J Biol Chem 272, 12116 - 12121 (1997)

22 Adams JL and Lee D: Recent progress towards the identification of selective inhibitors of serine/threonine protein kinases. Curr Opin Drug Discov Develop 2, 96 - 109 (1999)

23 Wang B, Lim DJ, Han J, Kim YS, Basbaum CB and Li JD: Novel cytoplasmic proteins of nontypeable Haemophilus influenzae up-regulate human MUC5AC mucin transcription via a positive p38 mitogen-activated protein kinase pathway and a negative phosphoinositide 3-kinase-Akt pathway. J Biol Chem 277, 949 - 957 (2002)

24 Duronio V, Scheid MP and Ettinger S: Downstream signalling events regulated by phosphatidylinositol 3-kinase activity. Cell Signal 10, 233 - 239 (1998)

25 Kane LP, Shapiro VS, Stokoe D and Weiss A: Induction of NF-kappaB by the Akt/PKB kinase. Current Biology 9, $601-$ 604 (1999)

26 Bierne H, Dramsi S, Gratacap MP, Randriamampita C, Carpenter G, Payrastre B and Cossart P: The invasion protein InIB from Listeria monocytogenes activates PLC-gammal downstream from PI 3-kinase. Cell Microbiol 2, 465 - 476 (2000)

27 Madge LA and Pober JS: A phosphatidylinositol 3-kinase/Akt pathway, activated by tumor necrosis factor or interleukin-1, inhibits apoptosis but does not activate NFkappaB in human endothelial cells. J Biol Chem 275, 15458 - 15465 (2000)

28 Gratton JP, Morales-Ruiz M, Kureishi Y, Fulton D, Walsh K and Sessa WC: Akt down-regulation of $\mathrm{p} 38$ signaling provides a novel mechanism of vascular endothelial growth factor-mediated cytoprotection in endothelial cells. J Biol Chem 276, $30359-30365$ (2001)

29 Derynck R and Feng XH: TGF- $\beta$ receptor signaling. Biochim Biophys Acta 1333, F105 - F150 (1997)

30 Derynck R, Zhang Y and Feng XH: Smads: transcriptional activators of TGF- $\beta$ responses. Cell 95, $737-740$ (1998)
31 Piek E, Heldin CH, Ten Dijke P: Specificity, diversity, and regulation in TGF- $\beta$ superfamily signaling. FASEB J 13, 2105 2124 (1999)

32 Massague $\mathrm{J}$ and Wotton D: Transcriptional control by the TGF$\beta /$ Smad signaling system. EMBO J 19, $1745-1754$ (2000)

33 Massague J: How cells read TGF- $\beta$ signals. Nat Rev Mol Cell Biol 1, $169-178$ (2000)

34 Hill CS: TGF- $\beta$ signalling pathways in early Xenopus development. Curr Opin Genet Dev 11, 533 - 540 (2001)

35 Roberts AB: The ever-increasing complexity of TGF- $\beta$ signaling. Cytokine Growth Factor Rev 13, 3 - 5 (2002)

36 Zhang Y, Feng XH and Derynck R: Smad 3 and Smad 4 cooperate with c-Jun/c-Fos to mediate TGF- $\beta$-induced transcription. Nature 394, 909 - 913 (1998)

37 Lopez-Rovira T, Chalaux E, Rosa JL, Bartrons R and Ventura F: Interaction and functional cooperation of NF- $\kappa \mathrm{B}$ with Smads. Transcriptional regulation of the junB promoter. J Biol Chem 275, 28937 - 28946 (2000)

38 Feng XH, Lin X and Derynck R: Smad 2, Smad 3 and Smad 4 cooperate with $\mathrm{Sp} 1$ to induce p15 (Ink4B) transcription in response to TGF- $\beta$. EMBO J 19, 5178 - 5193 (2000)

39 Wilckens T and De Rijk R: Glucocorticoids and immune function: unknown dimensions and new frontiers. Immunol Today 18, 418 - 424 (1997)

40 Wilckens T: Glucocorticoids and immune function: physiological relevance and pathogenic potential of hormonal dysfunction. Trends Pharmacol Sci 16, 193 - 197 (1995)

41 Karin M: New twists in gene regulation by glucocorticoid receptor: is DNA binding dispensable? Cell 93, 487-490 (1998)

42 Cato AC and Wade E: Molecular mechanisms of anti-inflammatory action of glucocorticoids. Bioessays 18, 371-378 (1996)

43 Webster JC and Cidlowski JA: Mechanisms of glucocorticoidreceptor-mediated repression of gene expression. Trends Endocrinol Metab 10, 396 - 402 (1999)

44 Takeuchi O, Hoshino K and Akira S: TLR2-deficient and MyD88-deficient mice are highly susceptible to Staphylococcus aureus infection. J Immunol 165, 5392 - 5396 (2000)

45 Sun H, Charles CH, Lau LF and Tonks NK: MKP-1 (3CH134), an immediate early gene product, is a dual specificity phosphatase that dephosphorylates MAP kinase in vivo. Cell 75, 487 - 493 (1993)

46 Groom LA, Sneddon AA, Alessi DR, Dowd S and Keyse SM: Differential regulation of the MAP, SAP and RK/p38 kinases by Pyst1, a novel cytosolic dual-specificity phosphatase. EMBO J 15, 3621 - 3632 (1996)

47 Imasato A, Desbois-Mouthon C, Han J, Kai H, Cato AC, Akira S and Li JD: Enhancement of NTHi-induced TLR2 expression by glucocorticoids via MKP-1-dependent inhibition of p38 MAPK. J Biol Chem 277, 47444 - 47450 (2002) 